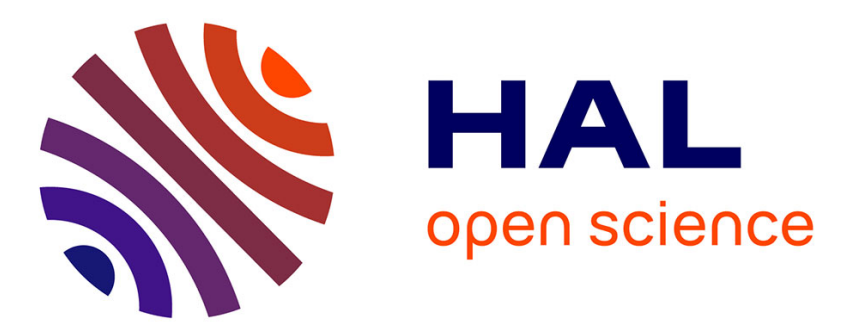

\title{
Une GPEC territoriale? De l'outil de gestion à l'institutionnalisation d'une nouvelle forme de GRH
}

Christophe Everaere, Catherine Glée

\section{To cite this version:}

Christophe Everaere, Catherine Glée. Une GPEC territoriale? De l'outil de gestion à l'institutionnalisation d'une nouvelle forme de GRH. Revue management \& avenir, 2014, n 73 , pp.73 à 91 . halshs-01073474

\section{HAL Id: halshs-01073474 \\ https://shs.hal.science/halshs-01073474}

Submitted on 9 Oct 2014

HAL is a multi-disciplinary open access archive for the deposit and dissemination of scientific research documents, whether they are published or not. The documents may come from teaching and research institutions in France or abroad, or from public or private research centers.
L'archive ouverte pluridisciplinaire HAL, est destinée au dépôt et à la diffusion de documents scientifiques de niveau recherche, publiés ou non, émanant des établissements d'enseignement et de recherche français ou étrangers, des laboratoires publics ou privés. 


\title{
Une GPEC territoriale ? \\ De l'outil de gestion à l'institutionnalisation d'une nouvelle forme de GRH
}

\author{
Christophe Everaere, Catherine Glée,
}

christophe.everaere@univ-lyon3.fr; catherine.glee@univ-lyon3.fr

Centre de recherche MAGELLAN. IAE Lyon

Université Jean Moulin Lyon3

Christophe Everaere est professeur des universités à l'IAE de Lyon, Université Jean Moulin. Membre du Centre de Recherche Magellan, ses recherches portent sur la gestion de l'emploi et des compétences individuelles et collectives. Dérivé de ses travaux initiaux sur la flexibilité, il vient de publier un ouvrage de synthèse sur les formes d'emploi et de travail atypiques ( atypiques. Quelles réponses au besoin de flexicurité ? », Préface de Hervé Sérieyx, Editions Liaisons Sociales, 2014).

Catherine Glée est Maître de conférences à l'université Jean Moulin Lyon 3, où elle dirige le master RHO (ressources humaines et organisation) de l'IAE Lyon; Elle est également responsable académique du MAE (Master Management Général) franco-polonais réalisé à Lodz (Pologne).

Membre du Centre de Recherche Magellan (IAE Lyon), ses recherches et enseignements portent sur les transitions professionnelles, les modes de management alternatifs, le management intergénérationnel.

Adresse postale : IAE Lyon, Université jean Moulin Lyon 3, 6 cours Albert Thomas, BP8242, 69355

Lyon Cedex 08 


\title{
Une GPEC territoriale ?
}

\section{De l'outil de gestion à l'institutionnalisation d'une nouvelle forme de GRH}

\section{Résumé :}

Dans un contexte d'incertitudes économiques et de précarité de l'emploi, la GPEC territoriale, visant à anticiper et mutualiser les pratiques de GPEC entre différents acteurs socioéconomiques d'un même bassin d'emploi, apparaît particulièrement opportune. Cependant, bâtir concrètement une GRH territoriale est difficile. A partir d'une recherche-action menée avec la Maison de l'Emploi et de la Formation de Lyon, nous essayons de comprendre les raisons de ces difficultés. Nous montrons qu'elles ne pourront être surmontées sans accepter d'entrer dans une démarche participative et anticipatrice qui facilitera le développement des interactions entre des hommes et des femmes partageant un projet commun.

Mots-clés : GRH, territoire, anticipation, mutualisation, institutions

\section{A territorial HRM ? From a management tool to the institutionalization of a new Human Resources Management.}

\begin{abstract}
:
In a context of growing unemployment, new HRM instrumentations outside organizations boundaries appear in order to help all the socio economic actors of a determined geographical area gathering and integrating their HRM policies.

However, in spite of a consensus on the importance and the interest to focus on this level of action for managing human resources, the instrumentation of a territorial HRM is emerging slowly and with a lot of difficulties. We try to analyze the reasons of these problems. In order to overcome these difficulties, we are pointing out the importance to help and to develop interactions between people who are sharing a common project
\end{abstract}

Key-words: collective human resources management, territory, institutions 
Dans un contexte de décentralisation de l'action publique appliquée au domaine de l'emploi et de la formation, de nouvelles instrumentations apparaissent à l'échelle du territoire.

Ainsi, depuis la loi du 13 aout 2004, relative aux libertés et responsabilités locales, «les régions coordonnent sur leur territoire les actions de développement économique des collectivités territoriales et de leurs groupements ». Le consensus obtenu entre le MEDEF ${ }^{1}$, $1^{\prime} \mathrm{UPA}^{2}$, la $\mathrm{CGPME}^{3}$ et les cinq organisations syndicales de salariés, qui a conduit à la signature de l'accord national interprofessionnel du 14 novembre 2008 sur la gestion prévisionnelle des emplois et des compétences, indique également cette centration sur le territoire et la pertinence de sa prise en compte pour promouvoir et coordonner de façon cohérente des politiques d'emploi et de développement des compétences. De même, depuis 2008, un fond national de revitalisation des territoires a été créé, mettant là encore l'accent sur le rôle stratégique du territoire, pour renforcer les politiques internes aux entreprises de gestion prévisionnelle des emplois et des compétences, ou, le cas échéant, pallier les insuffisances de ces politiques.

En effet, dans un contexte marqué par la précarité de l'emploi, la loi de cohésion sociale de 1998 (encore appelée loi Borloo) exprime clairement une volonté de décentralisation de l'action publique dans le domaine de l'emploi et de la formation professionnelle. A cela s'ajoute la réforme du dialogue social en 2004 et celle de 2008 qui, toutes deux, conduisent à appréhender de façon différente la question de la sécurisation des parcours professionnels. Il en résulte l'apparition de nouveaux outils de gestion des ressources humaines à l'échelle des territoires.

Parmi ceux-ci, le dispositif de GPEC Territorial, présenté dans la circulaire ministérielle du 29 juin 2010 relative au développement de la dynamique territoriale de gestion prévisionnelle des emplois et des compétences. Cette circulaire définit les «projets de GPEC territoriale » comme devant rassembler «sur la base d'un diagnostic partagé, autour d'un dialogue social territorialisé, (...) des acteurs socio-économiques locaux d'un bassin d'emploi concerné. Ils sont portés par des acteurs locaux, tels que les comités de bassin d'emploi, les maisons de l'emploi ou toute entité en mesure de fédérer les coopérations utiles ».

Ce glissement de la notion de GPEC à celle de GPECT (T pour territorial) exprime le besoin d'anticiper les mutations économiques que vivent au quotidien les entreprises sur ce nouvel espace d'action qui est le territoire et les expérimentations se multiplient prenant différentes formes ${ }^{4}$. Plus récemment encore, l'ANI du 11 janvier 2013 consacré à la compétitivité des entreprises et à la sécurisation de l'emploi, évoque directement des processus de mobilité inter-entreprises. Sans que le terme «territoire» ne soit évoqué, il est bien question d'envisager des formes de GRH qui débordent des frontières classiques de l'entreprise. L' article 7 de cet accord est ainsi formulé : Création d'un droit à une période de mobilité volontaire sécurisée dans les entreprises de plus de 300 salariés : à condition d'une ancienneté minimale de deux ans, les salariés peuvent travailler dans une autre entreprise pour changer d'emploi ou simplement travailler ailleurs, à leur initiative, et revenir ensuite dans l'entreprise d'origine (droit au retour) si l'expérience n'est pas concluante.

\footnotetext{
${ }^{1}$ MEDEF : Mouvement des Entreprises de France

${ }^{2}$ UPA : Union Professionnelle Artisanale

${ }^{3}$ CGPME : Confédération Générale du Patronat des Petites et Moyennes Entreprises

${ }^{4}$ II ne s'agit pas de faire une énumération exhaustive dans cette introduction mais on peut évoquer le projet TransverS'AL en Alsace, le projet MODEL 74, en Savoie, le projet Alliance en Rhône -Alpes qui, tous, visent à mettre en place une gestion territoriale des ressources humaines
} 
Sous la variété des appellations, il reste des objectifs identiques : comment apporter des réponses aux problèmes de mobilités professionnelles et de performances économiques, sur un bassin d'emploi donné, en mutualisant les ressources ? Comment dépasser les difficultés rencontrées par les entreprises pour mettre en œuvre une GPEC «intra-muros »? Comment concilier les aspirations des salariés à la sécurité et les exigences de flexibilité des entreprises ?

Ces objectifs se rejoignent autour de la notion délicate d'anticipation : Comment initier une démarche prospective qui permette de passer des actions souvent «à chaud » et uniquement curatives à des politiques globales, à la fois offensives et préventives ? Il y a donc de forts enjeux économiques qui expliquent la mobilisation des pouvoirs publics au niveau régional. Ainsi des Contrats Territoriaux Emploi Formation (CTEF) qui, mis en œuvre par l'Etat, sont une forme de convention spécifique de GPEC au niveau des branches présentes dans la région.

Or, curieusement, malgré un consensus sur l'importance et l'intérêt de cette démarche anticipatrice au niveau des territoires, comme en témoigne l'augmentation des accords signés, soit au niveau des branches, soit dans le cadre de la loi de cohésion sociale du 18 janvier $2005^{5}$, le nombre de réalisations réellement abouties reste limité. Les volumes d'emploi concernés par des mesures telles que le prêt de personnel interentreprises ou la mise à disposition (volontaire) des salariés préconisée par la loi du 30 décembre 2006 sur la sécurisation des parcours professionnels, sont faibles ${ }^{6}$. Le nombre et la variété des acteurs concernés par la GRH-T ne facilitent ni leur lisibilité, ni leur coopération et les difficultés de coordination qui en résultent entretiennent aussi des clivages (Bories-Azeau et Loubès, 2009). Sous la multiplicité des acteurs concernés qui pourraient faire «croire » à une forte structuration, l'instrumentation d'une GRH territoriale reste à construire et plusieurs éléments indiquent que cette construction sera difficile.

En conséquence, il nous semble intéressant d'étudier les raisons de ce décalage, de comprendre cette difficulté à opérationnaliser un instrument de gestion qui pourtant semble faire l'unanimité.

En nous appuyant sur un projet de recherche-action engagé en 2011 qui vise à développer une démarche prospective au niveau d'un territoire, via la mise en place d'un observatoire de l'évolution des emplois et des compétences sur le bassin d'emploi de la ville de Lyon (OECL), nous montrerons que les difficultés tiennent à la nature même du projet. En effet, ce dont il est question, selon nous, n'est pas de mettre en œuvre un outil, fréquemment présenté comme une «innovation sociale ». Il ne s'agit pas «d'un outil de plus » à la disposition des différents acteurs économiques. Ce qui est en question relève de l'institutionnalisation d'une nouvelle forme de management des ressources humaines qui, sortant des frontières spatiales de l'entreprise, conduit à mettre en place un nouveau mode de gouvernance et modifie ce faisant l'échelle décisionnelle, la nature des objectifs et la «philosophie » de fonctionnement.

\footnotetext{
${ }^{5}$ Parue au Journal Officiel du 19/01/2005, celle loi prévoit une obligation, pour toute entreprise de plus de 300 salariés, d'engager des négociations, tous les trois ans, en particulier, sur la mise en place d'un dispositif de GPEC ainsi que sur les mesures d'accompagnement associé.

${ }^{6}$ Quelques mois après avoir lancé en 2008, le programme " 6000 compétences 》 destiné à répondre aux besoins de recrutement des entreprises Airbus, STX et DNCS dans le bassin de Nantes Saint Nazaire, le conseil régional des Pays de la Loire a dû faire marche arrière devant le nombre et le niveau des difficultés à dépasser." Les élus au chevet de l’emploi », Liaisons Sociales Magazine, n 120, mars 2011.
} 
Pour appuyer notre démonstration, nous interrogerons, dans un premier temps, la notion de territoire. Cela afin de proposer une grille d'analyse des interdépendances qui s'y déploient, à l'aide des travaux issus du courant néo-institutionnaliste en particulier.

Dans un second temps nous présenterons notre terrain d'étude et les premiers résultats obtenus.

Nous conclurons sur l'idée de l'institutionnalisation d'une nouvelle forme de GRH dont l'état encore embryonnaire est masqué par des discours managériaux aussi séduisants que peu incarnés.

\section{Le territoire, nouvel espace d'anticipation et de management durable des ressources humaines?}

Le concept de GPEC-Territoriale fait débat (Culie et al., 2006 ; Bories-Azeau et al., 2008 ; Retour, 2008 ; Gazier, 2008) dans sa définition, dans ses champs d'actions, dans ses enjeux. La notion même de territoire est difficile à cerner du fait d'une variété de représentations que peuvent en avoir les différents acteurs, d'une polysémie liée aux différentes définitions que l'on peut en donner selon la perspective retenue. Il nous semble donc intéressant d'éclaircir cette notion. Nous rappellerons les différentes approches existantes ainsi que les perspectives conceptuelles relevées dans la littérature avant de proposer une définition «quadrilatère », c'est-à-dire fondée sur quatre dimensions.

\subsection{Différents prismes pour approcher la notion de territoire}

On peut approcher la notion de territoire sous différentes angles.

L'angle administratif et politique définit le territoire à travers le découpage lié aux différentes instances politiques (conseil général, régional...) et institutionnelles (Etat, départements, communes...). L'angle économique et social réalise une approche en termes de bassin d'emploi, de zones d'activité.....et met en lien la logique d'efficacité économique avec les besoins des populations évalués en termes de logement, revenus, déplacements et mobilités... L'angle sociétal et, pourrait-on dire, écologique, aborde le territoire en termes de lieu de vie, d'environnement, de relations de proximité. On peut retenir également l'angle historique davantage centré sur les notions de culture, de traditions, de coutumes qui donnent une identité à un territoire et souvent un ancrage de référence à ceux qui l'habitent. Enfin, l'angle géographique évoque un relief, une topographie, des ressources naturelles. Il y a ainsi à l'origine, l'idée d'un espace délimité géographiquement, séparé et d'une certaine façon protégé des autres espaces avoisinants. Cette notion d'espace à «préserver » est celle qui prévaut actuellement dans l'ensemble des actions mises en œuvre pour favoriser le développement économique en mobilisant les différents acteurs rassemblés sur même territoire. Mais si ces différents angles permettent de proposer une définition, penser la notion en la conceptualisant afin de mieux comprendre ce qui est à l'œuvre actuellement lorsque l'on parle de GPEC-T ou de GRH-T, est plus difficile.

Une première conceptualisation se fonde sur les «systèmes d'actions concrets 》 (Crozier, Friedberg, 1977 ; Friedberg, 1993) pour appréhender le territoire comme une structure de gouvernance territoriale (Bories-Azeau et Loubes, 2009). Cette perspective stratégique permet d'interpréter le territoire à travers des jeux d'acteurs, des mécanismes de coordination plus ou moins coopératifs qui permettent la mise en œuvre et le développement d'actions collectives. 
Dans cette perspective également la notion de réseaux, de densité et de pérennité du réseau (qui garantit la multiplicité, la rapidité, la pertinence et l'efficacité des échanges) se révèle cruciale (Granovetter, 1990).

On trouve une conceptualisation d'inspiration plus foucaldienne dans les travaux de Pesqueux (2007). Le territoire tel qu'évoqué dans la GRHT ou la GPECT est appréhendé comme une modalité, parmi d'autres, de " privatisation » d'un espace jusque là appartenant à et régi par l'Etat-Nation. Il apparaît fort à propos dans le contexte de mondialisation et d'une certaine façon en réponse à ce phénomène afin de remplacer le contrôle de type politique institué par l'Etat -Nation par un contrôle de type managérial développé au niveau du territoire. La référence actuelle à cette notion de territoire serait ainsi l'expression d'une idéologie de l'opportunisme. Là où l'Etat-Nation menait une «politique d'aménagement du territoire » à travers la DATAR ${ }^{7}$, le territoire évoqué aujourd'hui remplace la souveraineté politique de l'Etat par l'initiative des acteurs locaux œuvrant selon une logique organisationnelle c'est-àdire à travers la définition d'objectifs, fréquemment redéfinis au sein de stratégies ellesmêmes très évolutives puisque dépendantes des «opportunités et menaces » de l'environnement. L'autonomie des acteurs et le pouvoir qui leur est conféré sont aussi légitimés par la nécessité de s'adapter vite et du mieux possible. Le contrôle de type managérial se trouve ainsi considéré comme plus efficace que le contrôle de type politique. Selon cet auteur, c'est ainsi que la référence au territoire contribue à "l'idéologie de l'opportunisme actuel ».

Une conceptualisation que nous qualifierons d'économique se trouve présentée à la fin du XIXème siècle dans les travaux de l'économiste Marshall. Son ouvrage «Principes d'économie politique» (1890) met en évidence l'intérêt de la concentration de petites industries sur un espace géographique donné. Il s'agit d'un modèle économique à l'opposé de celui représenté par l'entreprise taylorienne et l'organisation fordiste. En effet, ces petites entreprises contribuent à développer une «atmosphère industrielle ». Leur regroupement volontaire permet la mutualisation et donc la création d'un effet de synergie également alimenté par un environnement propice à l'innovation, à l'apprentissage et à l'accès facilité à la main d'œuvre. Les districts industriels qui se développent en Italie du Sud dans les années 70 et 80 sont une illustration "grandeur nature » de ce concept marshallien de «localisation de l'industrie » avec interpénétration forte des entreprises et de la communauté (Becattini, 1992).

Cette idée d'interpénétration nous semble importante pour définir le territoire dans le cadre d'une GRHT ou d'une GPECT et nous conduit à proposer une lecture « quadrilatère » de la notion de territoire.

\subsection{Quatre dimensions pour conceptualiser la notion de territoire}

Quelles que soient les approches ou les définitions retenues, dans tous les cas, il est question de développement économique et sociale et un des acteurs essentiels de ce développement est l'entreprise, lieu de création de richesses ${ }^{8}$.

\footnotetext{
${ }^{7}$ Délégation à l'aménagement du territoire et à l'action régionale.

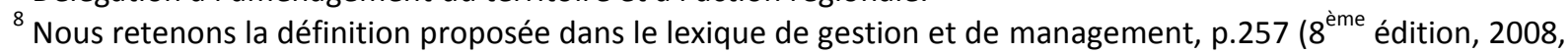
Dunod éditeur) selon laquelle l'entreprise est une unité économique autonome combinant divers facteurs de production, produisant pour la vente des biens et des services et distribuant des revenues en contrepartie de I'utilisation des facteurs.
} 
Nous privilégions, dans ce travail, une approche socio-économique en retenant le concept d'encastrement tel que défini par Polanyi (1944) puis Granovetter (1985). En effet, parler d'encastrement conduit à considérer l'entreprise non plus comme une boîte noire alimentée par des intrants et fournissant des extrants, déconnectée et isolée de l'ensemble de la société mais bien comme une entité ayant une réalité organique qui se construit, se développe, se transforme en fonction des réalités sociales et culturelles de son environnement et non pas exclusivement en fonction des exigences du marché.

Cette perspective socio-économique permet d'appréhender le territoire comme le lieu d'encastrement de l'entreprise, ce qui lui confère une réalité, une dynamique, un pouvoir.....et lui permet ainsi d'accéder à un statut propre. Il ne se réduit plus à un cadre neutre, aseptisé, réceptacle des actions et décisions prises en fonction de la seule logique marchande, mais devient un espace vivant qui permet d'institutionnaliser une nouvelle forme de GRH. C'est-àdire une gestion des ressources humaines déployée au niveau interentreprises et en fonction, non pas du seul objectif d'efficacité économique, mais du double objectif de sécurisation des parcours professionnels individuels et d'adaptation des entreprises aux exigences qui sont les leurs. Le territoire, lu à travers la grille néo-institutionnaliste, devient lieu d'encastrement de l'ensemble des actions menées, des décisions prises par les acteurs qui s'y trouvent, qu'il s'agisse des entreprises, des collectivités locales, de l'Etat à travers ses représentants, des individus. Il ne s'agit donc pas, dans cette perspective, d'un échelon intermédiaire entre le niveau micro où se trouvent les organisations et le niveau macro où officie l'Etat, mais d'un nouvel espace où des ajustements collectifs expriment une dynamique nouvelle à travers laquelle s'institutionnalisent des engagements mutuels. Le territoire devient ainsi le lieu où s'institutionnalise une manière d'être, de vivre ensemble, un espace où se façonnent des valeurs collectives sur la base de réalités multiples.

Mais là réside précisément une difficulté. Car comment construire un sentiment d'appartenance et une identité commune sur la base de la diversité des entités ? Comment dépasser la diversité de leurs attentes, contraintes, exigences, objectifs ? Cela met en évidence une deuxième dimension du territoire, sa dimension d'interdépendances.

Le deuxième élément de cette définition quadrilatère peut donc s'exprimer ainsi : le territoire s'appréhende comme un lieu d'interdépendances où évoluent des réseaux d'acteurs c'est-àdire des individus en interconnexions dont les relations et la forme de ces relations (qui peuvent être «fortes» ou «faibles» en densité et en quantité), sont une clef de compréhension de l'activité économique qui s'y déploie (Granovetter, 1973). Cette activité économique est encastrée dans des relations sociales, c'est à dire qu'elle ne peut se comprendre isolément du système de relations dans lequel elle se déroule, et cela fait de l'action économique une action sociale. Cette dimension d'interdépendances renforce donc la conceptualisation du territoire comme espace d'institutionnalisation d'une nouvelle forme de GRH.

Une troisième dimension qui poursuit cette approche institutionnaliste, consiste à appréhender le territoire comme un lieu de responsabilités partagées pour un management durable. En effet, concevoir le territoire comme lieu d'encastrement de l'action économique et comme lieu d'interdépendances conduit à parler de responsabilité sociale et sociétale. Chaque décision, chaque action a des conséquences pour l'ensemble de la communauté composée de «parties prenantes ». En analysant six cas de restructurations d'entreprises, Danièle Kaisergrüber (2006) met en évidence cette notion de responsabilité qui dépasse les frontières de l'entreprise pour s'étendre à l'ensemble du territoire. Conscientisée et assumée, la responsabilité permet d'entrer dans une logique de développement territorial cohérente où les 
interrelations entre l'ensemble des acteurs sont importantes, contribuent à créer une dynamique féconde grâce à la mobilisation « responsable » de toutes les parties prenantes.

Enfin, dernier élément du quadrilatère, l'appréhension du territoire comme un lieu d'anticipation. C'est, là encore, une difficulté car l'anticipation est différente de la prévision. Elle s'inscrit dans une démarche de prospective (Boyer, Scouarnec, 2009), plus subtile et délicate qu'un exercice de planification. La prévision renvoie à un exercice essentiellement quantitatif, basé sur la poursuite des tendances observées dans le passé. La planification, quant à elle, correspond à une démarche volontariste exercée en univers stable. L'anticipation, en revanche, correspond à une démarche de prospective c'est-à-dire une démarche où l'on se met en capacité d'aller dans une nouvelle direction en repérant des idées, des signaux faibles annonciateurs de ruptures fortes (Boyer et Scouarnec, 2009). Le futur devient ainsi autre chose que la reproduction du passé. Il émerge d'une imagination créatrice de l'avenir souhaitable avec la volonté de le préparer dès maintenant (Berger, 1964). Concevoir le territoire comme un lieu d'anticipation permet ainsi de passer d'une logique curative à une logique préventive, car dans ce cas il s'agit de fédérer les différents acteurs autour d'objectifs partagés. Ce qui prévaut est l'idée de faire advenir des projets forts, en développant des rapports de coopération qui permettront d'obtenir une performance globale tout en contribuant à une réelle promotion de l'homme (Berger, 1964).

En définissant le territoire comme un lieu d'encastrement de l'entreprise dans une réalité économique, sociale, écologique; un lieu d'interdépendances entre réseaux d'acteurs multiples; un lieu de responsabilité sociale et sociétale partagée ; un lieu d'anticipation où s'exerce une démarche prospective à l'opposé de la démarche planificatrice, la GPEC qui s'y déploie n'est plus seulement un outil de gestion, certes représentant une innovation sociale, mais seulement un outil «de plus ». Elle relève davantage de l'institution d'un «vivre ensemble ».

Mais «vivre ensemble en société » est difficile lorsqu'il y a, comme c'est le cas sur un territoire, multiplicité des représentations, des enjeux et des aspirations. Le projet que nous présentons maintenant est une illustration de cette difficulté qui semble confirmer le caractère « institutionnalisant » de la démarche GPEC-T.

\section{La création d'un observatoire pour terrain d'études}

\subsection{Un projet de recherche - action original, aux enjeux stratégiques}

L'opération menée avec la Maison de l'Emploi de Lyon pour élaborer un Observatoire de l'Evolution des Emplois et des Compétences de la ville de Lyon (OECL) est en phase de démarrage. La construction de cet observatoire fait l'objet d'une coopération relativement originale entre la Maison de l'Emploi et de la Formation (MDEF) de la ville de Lyon, des universitaires engagés sur des sujets articulant les flexibilités et la sécurisation des parcours professionnels, des étudiants de master en GRH et enfin l'ANDRH du Rhône.

Il s'agit d'un projet de recherche-action tel que définit par les auteurs Coghlan et Brannick (2005) ; Eden et Huxham (1996) et Robson (2002). 
Ces auteurs mettent en évidence les différentes dimensions d'une recherche-action également présentes dans notre projet :

Il s'agit d'une recherche engagée dans l'action et non pas sur l'action (Coghlan et Brunwick, 2005); L'implication des différents protagonistes (universitaires et praticiens) est de forme coopérative. A ce propos, Coghlan et Brunwick (2005) parlent de l'importance de la forme démocratique de ce travail de partenariat. Elle s'est traduit dans notre cas par des séances de travail nombreuses et régulières (rencontre mensuelle alternativement dans les locaux de la Maison de l'Emploi et à l'Université durant toute la durée de l'étude) qui réunissait systématiquement les universitaire auteurs de ces lignes et les membres de l'équipe projet de la Maison de l'Emploi fédérée autours du directeur de la MDEF Rhône ainsi qu'un représentant de l'ANDRH et un représentant des étudiants du master ; le processus de recherche est de nature itérative (Robson, 2002). Dans notre cas, en effet, la stratégie de recherche s'élaborait «chemin faisant» en fonction des réponses (ou non réponses) des entreprises contactées, en fonction des connaissances glanées lors de témoignages, de lectures, en fonction des premiers résultats obtenus et des débats au sein du groupe de recherche - action. Les implications sont réelles et fortes en termes de transferts de connaissances utiles à la fois à l'action et à l'enrichissement et l'éclaircissement de questions théoriques (Eden et Huxham, 1996), ce que montrent l'objectif et les enjeux ci-dessous présentés.

L'objectif est de permettre aux acteurs locaux, institutionnels et économiques, de mieux anticiper les mutations économiques du territoire (ici délimitée au bassin d'emploi de la ville de Lyon). Derrière cet objectif ambitieux, il s'agit d'anticiper le besoin (en volume et en nature) des compétences nécessaires au fonctionnement et au développement des organisations au sens large (employeurs publics et privés) d'un territoire. Cela constitue un enjeu majeur pour les employeurs. Mais c'est aussi un enjeu pour de nombreux autres acteurs du territoire, en particulier les structures chargées de former et préparer les futurs citoyens détenteurs des compétences (ou qualifications) dont les entreprises ont besoin.

Un autre enjeu à très fort contenu problématique réside dans la capacité et la volonté des acteurs du territoire de mutualiser leurs réflexions et efforts pour analyser et anticiper ensemble - les besoins et les ressources d'emplois et de compétences.

Ce projet de recherche-action contribue donc à réunir une pluralité d'acteurs (privés / publics ; privés intra et inter secteurs d'activités) présents sur un même territoire pour agir ensemble dans une logique gagnant-gagnant, afin de :

$>$ sécuriser le parcours professionnel des individus de ce territoire ;

$>$ faciliter le recrutement des entreprises en besoin de compétences ;

$>$ faciliter les départs des collaborateurs des entreprises en phase de réduction d'effectif par des retours plus rapides à l'emploi dans les entreprises voisines ;

$>$ optimiser l'efficacité des services publics de l'emploi et des structures d'insertion ;

$>$ mieux repérer et anticiper les besoins en qualification pour les organismes de formation de ce territoire.

\subsection{Choix méthodologiques et premiers résultats : un tableau mitigé.}

Dans cette première étape, la méthodologie retenue est exploratoire. Nous avons réalisé entre mai et juillet 2011 une série d'entretiens semi-directifs auprès d'une dizaine de DRH d'entreprises lyonnaises pour connaître leur pratique de GPEC et appréhender leur degré de sensibilité à une éventuelle démarche de GRH territoriale. 
La variété sectorielle des répondants est forte, à la fois en terme de secteur d'activité et de taille. Les effectifs varient de 80 à 5000 salariés (sur l'ensemble du territoire national avec une organisation multi-site dont le siège est basé à Lyon pour l'une des entreprises). Les secteurs d'activités représentés sont les suivants : industrie métallurgique aéraulique, conseil en ingénierie et organisation industrielle, installation et vente de panneaux photovoltaïques, travail intérimaire, un centre de gérontologie, une usine de conception et injection de bouchon en plastique, une société de maintenance et travaux industriels nucléaire, un office public de l'habitat, et une société de transport express de colis et documents.

Cette variété sectorielle était souhaitée. En effet, l'objectif de l'observatoire de l'évolution des emplois et des compétences de Lyon est de permettre une analyse du degré de transférabilité des emplois et des compétences entre les entreprises du territoire. Si tous les secteurs d'activité d'un territoire donné sont en déclin, une GPEC territoriale ne présente aucun intérêt, sauf à organiser collectivement les complaintes de chacun ou les « obsèques » économiques et sociales du territoire en question s'il est définitivement condamné. En revanche, si le territoire est composé de secteurs d'activités relativement différents qui connaissent des cycles opposés, alors des flux d'emploi deviennent envisageables et sûrement nécessaires, à la fois pour fournir en ressources humaines les entreprises du secteur qui se développent sur le territoire. Et aussi pour fournir en emploi et en revenus les habitants du territoire touchés par les difficultés économiques de leur entreprise ou secteur d'origine qui peut être amenée à devoir les licencier par défaut de commande, d'activité, de changement technologique, peu importe les raisons. Dans ce cas, une GRH-T peut apporter toute son utilité, en permettant de repérer des passerelles possibles pour passer d'un secteur d'activité, d'une entreprise ou d'un métier à un autre, mais sans devoir déménager. Cette démarche renvoie évidemment à la question de la sécurisation des parcours professionnels. Mais cette démarche de sécurisation profite autant aux habitants du territoire qu'aux entreprises. Il y a ainsi l'opportunité d'entrer dans une logique gagnant-gagnant dans l'intérêt commun de tous les acteurs du territoire.

Au-delà de leur activité, les entreprises sont aussi diverses dans leur ancienneté. Si une majorité d'entreprises sont de création plutôt récente (5 structures sont nées entre 1980 et 2007), on compte aussi des entreprises plus anciennes : 3 créées entre 1920 et 1945. Le point commun à tous les répondants est leur présence sur le bassin d'emploi de Lyon et leur appartenance au réseau ANDRH du Rhône grâce à qui nous sommes entrés en contact avec les DRH.

Cette exploration a été menée sous forme d'entretiens semi-directifs (King 2004 ; Mucchielli, 2009 ; Blanchet, Gotman, 2010) réalisés en tête à tête avec les DRH des entreprises et des binômes d'étudiants du Master de RHO (certains binômes étaient mixtes enseignant-étudiant).

Un guide d'entretien a donc été réalisé qui organisait les thèmes abordés de la façon suivante :

Le premier regroupement de questions était de nature descriptive :

- activité(s) de l'entreprise, ancienneté, implantation géographique, situation économique globale (état du marché : porteur ou en déclin),

- structure des effectifs : nombre de salariés en ETP, âge moyen, classement par âge, genre, catégories professionnelles, métiers, niveaux de qualification, types de contrats (intérimaires notamment), taux de turnover.

Un second regroupement de questions portait sur les stratégies de recrutement en lien avec les éventuels besoins de flexibilité, la politique de formation et de mobilité de l'entreprise. 
Dans un troisième temps, les questions portaient sur l'existence ou non d'un accord GPEC d'entreprise ou de branche. Si un accord était signé, quelles en étaient les orientations prioritaires (recrutement, mobilité interne, politique de formation, transmissiondéveloppement des compétences) ? Seules deux entreprises sont concernées par un accord de branche (travail temporaire et métallurgie), lesquelles ont par ailleurs signé un accord de GPEC interne. Pour les autres, c'est principalement l'approche du seuil des 300 personnes (seuil à partir duquel un accord est obligatoire) qui les amène à y réfléchir.

Les résultats, à ce niveau, confirment le phénomène déjà repéré par ailleurs d'une faible diffusion de la GPEC qui se traduit par la rareté des accords : «Seulement 16\% des salariés appartenant à une entreprise soumise à l'obligation triennale de négocier sont concernés par un accord et moins de $2 \%$ des entreprises soumises à l'obligation ont signé un accord ${ }^{9}$ ».

Les questions posées ensuite portaient sur l'analyse des métiers en lien avec l'évolution quantitative et qualitative de l'activité. Les répondants étaient invités à classer et chiffrer les emplois de leur entreprise selon les catégories suivantes (en fournissant si possible des éléments d'explication) :

- Emplois stratégiques: emplois pour lesquels l'entreprise doit assurer la pérennité de son savoir-faire et de ses compétences / emplois clés pour le développement de l'entreprise?

- Emplois à recrutement difficile et quelles solutions envisagées pour parer à ces difficultés ? : emplois pour lesquels l'offre de main-d'œuvre est réduite sur le marché et métiers nécessitant une période longue d'apprentissage?

- Emplois émergents : emplois nouveaux, avec des besoins significatifs ?

- Emplois à évolution significative de compétences: emplois à évolution importante de périmètre de compétences, à quel horizon ?

- Emplois ayant vocation à disparaître : emplois qui, du fait des transformations de l'entreprise, ne sont plus porteurs d'avenir au sein de l'organisation, à quel horizon?

- Emplois ayant vocation à être externalisés / sous-traités

- Quels projets d'embauche et pour quelles compétences ?

L'entretien tournait enfin autour de la GPEC Territoriale avec l'objectif de savoir si l'entreprise, par le biais de son (sa) DRH, connaissait et/ou avait recours à des dispositifs de type : prêt de main d'œuvre inter entreprise à but non lucratif, groupement d'employeurs, plateforme territoriale de reclassement, bourses de $\mathrm{CV} /$ compétences interentreprises, etc.). Deux questions précises permettaient d'aborder frontalement la question de l'entreprise dans son territoire et ses réseaux :

- Pensez-vous qu'une coopération interentreprises soit possible pour régler cette question de GPEC au niveau territorial? Si oui, comment, à quelles conditions ? Seriez-vous prêt à vous engager dans ce type de projet ? Si non : pourquoi?

- Pensez-vous qu'une coopération inter-acteurs (entreprises, acteurs institutionnels, ...) sur le marché de l'emploi soit possible pour régler cette question de GPEC au niveau territorial? Si oui, comment, à quelles conditions ? Seriez-vous prêt à vous engager dans ce type de projet ? Si non : pourquoi?

\footnotetext{
${ }^{9}$ X. Baron, A la rescousse des territoires, Metis, correspondances européennes du travail, 2010.
} 
Ce sont les réponses à ces deux questions qu'il nous semble intéressant d'analyser dans le cadre de cette réflexion sur la GPEC territoriale. Elles permettent de réaliser un classement en deux groupes d'entreprises : un groupe que nous avons qualifié de sceptiques et un groupe d'entreprises que nous avons qualifié d'actifs.

\section{$\underline{\text { Arguments des sceptiques concernant la GPEC-T: }}$}

Les sceptiques sont composés de DRH qui pensent qu'avant d'aller vers de la GPEC Territoriale, il est d'abord nécessaire d'avoir une GPEC interne solide ${ }^{10}$, ce qui est loin d'être le cas de toutes les entreprises. Cette remarque semble indiquer qu'une GPEC territoriale peut compléter une démarche de GPEC interne, mais qu'elle ne peut pas s'y substituer ou remplir un «vide », dans le cas où les bases mêmes d'une GPEC interne font défaut.

L'un des répondants «sceptique » a insisté également sur la nécessité d'avoir une description fine des métiers pour permettre une analyse des compétences qui seraient transférables d'une entreprise (ou secteur) à un(e) autre. Les intitulés génériques de métiers sont effectivement parfois trompeurs. Une discussion engagée par deux DRH présents à la réunion de restitution de ces premiers résultats a permis de montrer que le métier de soudeur dans le secteur de la tôlerie-aéraulique, mobilise des compétences différentes de celles d'un soudeur dans le secteur de la chaudronnerie tuyauterie en milieu nucléaire.

A contrario, il existe des métiers relativement "génériques » (comptable, assistante de direction, informaticien, contrôleur de gestion, acheteur, logisticien, responsable marketing, des ressources humaines, etc.), autant de métiers que l'on trouve généralement dans des fonctions dites de support pour lesquels la mobilité intersectorielle est probablement plus facile que pour des métiers spécifiques situés au « cœur du métier » de l'entreprise.

Un répondant «sceptique » a énoncé une autre réserve relative à la GPEC-T qui est celle de la crainte de partager des informations confidentielles ou des compétences avec des entreprises qui seraient directement concurrentes sur les mêmes marchés.

Une dernière réserve formulée concernant les démarches de mutualisation des compétences est celle liée à des différences possibles au sein des entreprises en matière de conventions collectives et d'acquis sociaux. Cette difficulté est plus marquée pour des dispositifs de mobilité provisoire entre entreprises, comme dans le cas du prêt de personnel inter-entreprises à but non lucratif. Démarche qui s'apparente au principe des «vases communicants » selon la formule de Jean Pierre Anciaux, député de Saône et Loire et promoteur avec Bernard Gérard (député du Nord) et Jean Frédéric Poisson de la loi du 9 juin 2009 concernant le prêt de personnel interentreprises à but non lucratif dans l'optique «d'encourager la mobilité professionnelle ».

\section{Des répondants qualifiés d'actifs en matière de GPEC - Territoriale}

L'un des répondants est déjà impliqué sur le territoire de Lyon dans un dispositif qui s'apparente à une GPEC «extra-muros ». Plusieurs entreprises lyonnaises sont en effet engagées dans un projet appelé «Alliance» qui vise à permettre aux salariés de ce groupement d'emprunter des «passerelles sécurisées » leur permettant de découvrir un autre environnement professionnel à des fins d'élargissement des compétences, de valorisation

\footnotetext{
10 Une GPEC qualifiée de solide correspond à une entreprise qui serait en mesure d'apporter des réponses relativement précises à l'ensemble des questions posées dans le cadre de l'enquête.
} 
d'expertise ou de reconversion. Ce dispositif s'apparente à une forme de détachement sécurisé d'une entreprise à une autre, comme cela se pratique dans la fonction publique.

Un autre répondant qualifié d'actif a mentionné une bourse d'emploi correspondant à des offres d'emplois émanant d'entreprises du territoire qui sont regroupées sur un même site Internet.

Un groupement de moyens de gestion (GMS) a été évoqué par l'un des répondants. Il s'agit en l'occurrence pour plusieurs structures de soins de la ville de Lyon de mutualiser des compétences qui travaillent le matin dans une structure donnée, et l'après-midi dans une autre. A noter que cette pratique s'inscrit dans un contexte global de pénurie de compétences particulièrement aigu dans le secteur médical. On constate donc ici une forme de partage ou de gestion en commun de RH poussée par une pénurie de compétences sur le territoire en question. Le même répondant est par ailleurs engagé dans une démarche de création d'un référentiel métier commun au sein de la fonction publique hospitalière. Des offres d'emplois et des $\mathrm{CV}$ de candidats non retenus sont également diffusés entre structures de soin du territoire. Des investissements formation sont également partagés entre différentes structures pour des formations identiques. En l'occurrence, selon l'adage nécessité fait loi, la pénurie de compétences relativement chronique dans le secteur de la santé et les contraintes de restriction budgétaire semblent avoir poussé différents employeurs du territoire lyonnais de ce secteur d'activité à se concerter, à partager des RH et des dépenses de formation. On peut également ajouter que la problématique concurrentielle joue moins dans ce secteur que pour des entreprises privées du secteur marchand.

Nous aurions souhaité trouver une ligne de démarcation claire entre les entreprises dites sceptiques et les entreprises dites actives en matière de GPEC-Territoriale, notamment autour des facteurs de contingence classiques que sont la taille de l'organisation, le niveau de complexité de l'activité, le clivage privé / public, etc. Mais compte tenu de la taille limitée de notre échantillon, et de ce que nous donnent à entendre les propos de nos interlocuteurs en lien avec le domaine d'activité de leur entreprise, rien ne ressort permettant d'attribuer telle opinion (plutôt sceptique $v s$. plutôt actif) émise, à un facteur explicatif objectif clair.

\section{De la difficulté de mutualiser et d'anticiper « ensemble »}

$\mathrm{Au}$ final, les expériences concrètes pouvant s'apparenter à de la GPEC Territoriale sont modestes à la fois dans les dispositifs eux-mêmes: bourse de l'emploi, formes de détachement sécurisé de salariés entre différentes entreprises du territoire (projet Alliance, présenté plus haut), mutualisation de compétences; et aussi dans le nombre de salariés concernés par de la mobilité professionnelle inter-entreprises du dispositif Alliance, par exemple ou celui de la mutualisation de RH entre structures de soins.

En regardant plus attentivement les dispositifs concrets évoqués par les répondants qualifiés d'actifs, on peut se demander si les dispositifs évoqués relèvent vraiment d'une démarche de GPEC, en insistant sur le volet prévisionnel et anticipé de la démarche ? Pour la bourse de l'emploi, il est question de mettre en commun sur un même site Internet des offres d'emploi d'entreprises voisines. Est-ce de la GPEC - territoriale ? Il est permis d'en douter.

Une difficulté importante dans ces intentions et démarches de GPEC-T réside dans la signification de la lettre $\mathrm{P}$. Il est question de prévoir (gestion prévisionnelle...), donc à une échelle de temps qui devrait être significative pour permettre de préparer des évolutions 
professionnelles, des mutations, des transitions, des reconversions, des formations, des transferts de savoir suite à des départs à la retraite de personnes détentrices de compétencesclés pour l'entreprise, par exemple. Un horizon d'anticipation d'au minimum 3 ans est souvent cité pour faire une GPEC qui présente un intérêt. Or, l'horizon temporel des prévisions en entreprise est parfois très court : "Notre visibilité est nulle au-delà d'un trimestre... nous faisons des prévisions chiffrées, ce sont des références nécessaires, mais l'expérience a montré que ces chiffres sont rarement confirmés ! ${ }^{11}$. Comment anticiper sérieusement face aux incertitudes de toute nature (économique, politique, réglementaire, financière, technologique, etc.) qui pèsent sur de nombreux marchés et de nombreuses entreprises ?

Pour les dispositifs de détachement sécurisés inter-entreprises, les salariés peuvent explorer de nouvelles opportunités professionnelles dans des entreprises voisines et partenaires de l'opération. Mais est-ce de la GPEC - territoriale ? Une fois de plus, il est permis d'en douter. Il en est de même pour la mutualisation de compétences qui semble davantage répondre à un manque chronique de compétences disponibles sur le marché de l'emploi qu'à une démarche concertée et partagée de GPEC entre différents employeurs d'un même territoire.

Le constat est identique chez d'autres auteurs. Difficile, par exemple, de ne pas voir l'expérience de GTEC au sein de Arves Industries comme un échec : «l'outil est figé », diton sobrement ${ }^{12}$. Il est expliqué que la difficulté provient de la composition des partenaires dont les enjeux sont trop différents. Et qu'en même temps, les entreprises étant concurrentes entre elles, donc identiques ou proches d'un point de vue activité, elles refusent de jouer le jeu du partage des informations et de la mutualisation des ressources humaines. Les acteurs sont trop différents ou trop semblables ? La compréhension des causes de l'échec ou de la suspension du dispositif n'est manifestement pas simple.

Face à ces constats, les discours de toutes parts insistent sur l'importance et l'intérêt d'animer des marchés locaux du travail, de «territorialiser » les GPEC, de développer des formes multiples de gestion locale de l'emploi et des compétences, d'assurer un développement maîtrisé des mobilités professionnelles.... Territoire, proximité, mutualiser, anticiper... Tous ces concepts sont séduisants et recueillent une adhésion de principe généralisée. Pourquoi dès lors les expériences concrètes de GRH-T sont aussi marginales ?

Il est dit que les Maisons de l'Emploi (MDE) se voient chargées du " développement de la gestion territorialisée des ressources humaines » (Bories-Azeau et Loubès, 2009). Mais que gèrent - effectivement - les MDE ? N'y a-t-il abus dans l'usage du verbe « gérer »? N'est-ce pas l'une des limites de ce concept séduisant de GRH-T que de constater l'existence de très (trop ?) nombreux acteurs concernés par l'emploi et les compétences présents sur les territoires, mais qui n'ont finalement qu'une capacité et légitimité d'action en matière de GRH qui n'est que marginale, voire symbolique : observer, anticiper, constater (notamment les écarts entre l'offre et la demande de travail sur les territoires), certes...; mais pas «gérer» les ressources humaines d'un territoire... Seul un employeur exerce cette fonction et cette responsabilité de «gestion» des ressources humaines.

\footnotetext{
${ }^{11}$ Propos d'un DRH cité dans le rapport de H. Rouilleault, « Anticiper et concerter les mutations. Rapport sur l'obligation triennale de négocier le dispositif de GPEC », La Documentation Française, 2007, p. 45.

12 "L'outil [base de donnés RH accessible par Internet] est peu à peu contesté par les différents groupes d'acteurs. Le réseau n'est à ce jour pas encore stable. Les entreprises n'utilisent pas l'outil GTEC. Les financeurs du projet émettent un avis plutôt mitigé quant aux résultats atteints ; les consultants ont terminé leur mission et transfère l'outil au pôle » (I. Mazzili, op. cit. p. 18).
} 
A ce sujet, il existe des structures délibérément créées pour faire de la GRH - T. Il s'agit des groupements d'employeurs (GE) ${ }^{13}$. Or, au vu des chiffres et malgré l'ancienneté de ce dispositif (créé en 1985), les groupements d'employeurs n'ont jamais pris d'ampleur (moins de 40000 personnes concernées ${ }^{14}$, contre plus de 2 millions d'intérimaires en données brutes). Pourtant, les GE sont réputés sécurisants pour les personnes puisque le GE a vocation à recruter sur des emplois stables (contrats à durée indéterminée - CDI).

Un rapport de l'IGAS portant sur le prêt de personnel dans un pôle de compétitivité (Minalogic, en l'occurrence) fournit quelques données statistiques sur l'ampleur de ce dispositif basé également sur le principe d'une GRH-T. Le nombre de personnes concernées par ce dispositif, pourtant souple pour les entreprises et sécurisant pour les salariés, est extrêmement faible : quelques centaines de personnes tout au plus. «Les prêts de personnel entre membres d'un pôle de compétitivité sont finalement restés très peu de chose (une petite quarantaine entre 2007 et la rédaction du rapport en 2010) au regard des dizaines de milliers de mises à disposition pratiquées en permanence dans le cadre de projets de recherche et développement par le biais des contrats de prestations de services qu'assurent les sociétés d'ingénierie-conseil, sans fléchissement trop marqué, du reste, depuis le début de la crise de l'emploi » (Guthmann et al., 2010, p. 5).

Concernant l'expérience de MODEL 74 à Annecy, fin 2011, après 18 mois, le bilan est jugé en demi-teinte : 25 entreprises s'impliquent dans l'expérience de prêt de personnel interentreprises à but non lucratif, 25 salariés ont été détachés sur les 57 inscrits dans la démarche.

A Lyon, les ordres de grandeur sont relativement identiques : fin juin 2013, soit 2,5 ans après le démarrage de l'opération, 17 structures ont adhéré au dispositif MODEL 69 (des entreprises privées de taille et secteur d'activité divers, ainsi que des mairies, une structure associative et des organismes de formation). Parmi les 17 structures, 10 ont présenté des salariés volontaires au détachement et 13 ont été mises en relation pour un poste à pourvoir. Une bourse à l'emploi a répertorié à un instant t (fin juin 2013) 60 postes à pourvoir dans des fonctions diverses : services généraux, production, bureaux d'études, postes sur des chantiers. Sur les 2,5 ans d'existence du dispositif, 85 salariés ont bénéficié du prêt de personnel interentreprises : ces salariés ont tous rencontré individuellement la coordinatrice Model69 afin de répondre à leurs questions et s'assurer de leur volontariat. 77 d'entre eux ont confirmé leur intérêt et ont été accompagnés par un des 3 cabinets RH "labellisés Model" afin d'élaborer leur passeport compétences. En fonction de leur compétences et projet, ils ont été mis en relation avec des entreprises intéressées par leur profil. Le bilan mené fin juin 2013 note également que sur les 41 salariés volontaires les plus récents, 25 ont arrêté leur

\footnotetext{
13 « Un groupement d'employeurs est une structure qui réunit plusieurs entreprises. Cette structure peut être constituée sous forme associative (association loi 1901), ou sous forme de société coopérative. Le but du groupement d'employeurs est de recruter un ou plusieurs salariés et de le(s) mettre à disposition de ses membres, selon leurs besoins. Il peut également apporter à ses membres son aide ou son conseil en matière d'emploi ou de gestion des ressources humaines. Le GE favorise la stabilité des salariés dans leur emploi en leur offrant de travailler dans plusieurs entreprises regroupées sur un même territoire. Le groupement est ainsi un moyen efficace de fixer une main-d'œuvre dans un bassin d'emploi, notamment en zone rurale. " (extrait du site Internet du Ministère de l'Emploi, du Travail, de la formation professionnelle et du dialogue social, dernière mise à jour janvier 2013).

${ }^{14}$ Hors secteur agricole, il y aurait en France environ 400 GE regroupant 10000 entreprises adhérentes, qui emploient 12000 personnes. Ce chiffre atteint 35000 salariés si l'on prend en compte l'ensemble des 4500 $\mathrm{GE}$, tous secteurs confondus, pour un chiffre d'affaires global de 650 millions d'Euros
} 
accompagnement individuel en cours de route car leur entreprise avait retrouvé de l'activité et a stoppé net ce projet de détachement ${ }^{15}$.

La nouveauté de ce dispositif pourrait servir à expliquer sa faible diffusion. Mais l'argument ne tient pas. L'auto-entreprenariat est presque aussi récent que le prêt de personnel (la $1^{\text {ère }}$ loi concernant le prêt de personnel interentreprises à but non lucratif date du 9 juin 2009). Le régime de l'auto-entreprenariat a été créé en août 2008 (dans le cadre de la loi de modernisation de l'économie), avec le décret d'application paru en janvier 2009. Mais ce sont près de 900000 auto-entrepreneurs qui se sont inscrits (même s'ils ne sont actifs que pour la moitié d'entre eux), tandis que les salariés prêtés entre différentes entreprises d'un territoire ne se comptent qu'en centaine(s) d'unités.

Et pourtant ! Face à ces constats plutôt décourageants, il y a la "conviction » de certains hommes et femmes de terrain que «l'approche par les territoires est celle qui répond le mieux aux grandes problématiques actuelles » (Fromantin, 2012, p.6). Car cette approche permet de créer une dynamique ascendante, véritable fondement d'une requalification de chaque sujet menée selon les principes d'initiative et de responsabilité. C'est aussi une approche qui permet de combiner une vision prospective et des mesures précises au quotidien. Il nous importe de conclure notre réflexion en mettant en évidence des pistes d'action managériales.

\section{Conclusion}

Ce qui semble faire défaut dans les groupements d'employeurs ou dans le prêt de personnel n'est pas le manque de sécurisation pour les personnes. Les personnes prêtées ou mises à disposition par les GE conservent leur CDI et leur rémunération. Seul le lieu d'exercice du travail change car il s'exerce à différents endroits du territoire (chez les adhérents du GE ou entre les entreprises du bassin d'emploi qui se prêtent leurs RH). En ce sens, le GE ainsi que le prêt de personnel échappent à la critique de précarité à laquelle les formes de travail atypiques sont souvent associées.

En revanche, le processus de mutualisation auquel renvoie le GE ou le prêt de personnel est contraignant pour les entreprises. Ces dispositifs nécessitent en effet une certaine préparation et anticipation.

Or, ce besoin d'anticipation pour définir le cadre de la mutualisation ou du partage des RH entre plusieurs entreprises, est en contradiction direct avec le besoin de réactivité, c'est à dire le besoin de pouvoir disposer très rapidement de quelqu'un en cas de surcroît temporaire d'activité, ou pour remplacer un salarié absent pour cause de maladie, le matin même parfois où cette absence se manifeste et met en difficulté l'entreprise concernée. Dans cet enjeu de réactivité, l'intérim est imbattable ; les GE, de leur côté, de par leur objectif premier de mutualisation, ne peuvent pas lutter sur l'argument de la réactivité. Or, les entreprises ont bien davantage besoin de réactivité que de mutualisation.

La problématique de mutualisation inhérente aux GE ou au prêt de personnel nécessite une gestion concertée d'une même ressource entre plusieurs structures. Il faut analyser en commun les compétences des personnes en question, s'assurer qu'elles soient relativement similaires tout autant que les conditions de travail. Il faut définir les périodes de mises à

\footnotetext{
15 A. Marsick, "Comment le prêt de personnel questionne et s'intègre dans les politiques RH des entreprises ?», mémoire de master RHO, IAE Lyon - Université Lyon 3, septembre 2013.
} 
disposition ou de prêt, s'assurer que les disponibilités soient compatibles (pas au même moment). Il faut envisager les possibilités de non-retour ou d'embauche par l'un des adhérents, traiter les contraintes de distance même si on reste dans un bassin d'emploi, informer les IRP, préparer les avenants au contrat de travail dans le cas du prêt de personnel, etc. Dans ce contexte, il est donc indispensable d'anticiper, de préparer, de se concerter, se coordonner ; or, ce temps de préparation et de coordination empêche les dispositifs d'hyper réactivité.

Les contraintes sont donc très différentes de celles demandées à une entreprise de travail temporaire dont les clients n'ont pas besoin de se concerter pour « partager » une même compétence. La problématique du partage est uniquement du ressort de l'entreprise de travail temporaire. C'est l'affaire de celle-ci que d'optimiser la gestion de « ses » intérimaires. Nul besoin pour les entreprises clientes de se concerter et de se coordonner dans l'intérim. A la différence du GE ou du prêt de personnel qui nécessitent un travail de connaissance mutuelle et un effort de partage d'une même ressource humaine qui peut s'avérer particulièrement délicat si les entreprises en question exercent dans le même domaine d'activité et peuvent donc se retrouver en concurrence frontale.

Il est donc très important de cerner l'objectif majeur de la GRH-T :

- soit la recherche d'une flexibilité et d'une capacité d'adaptation très rapide et facilement réversible de la main d'œuvre à des variations de charge relativement imprévisibles. C'est le domaine d'excellence des entreprises de travail temporaire.

- soit, la recherche de mutualisation pour partager, entre plusieurs entreprises, les mêmes ressources, au même moment et à temps partiel dans le cas des groupements d'employeurs, ou de façon alternée et à temps plein dans le cas du prêt de personnel.

Les deux options ou les deux besoins semblent disjonctifs : c'est l'un ou l'autre. La mutualisation ne peut se situer dans la réactivité, parce que la mutualisation nécessite de la préparation, quand la réactivité induit une réponse très rapide. Mais la mutualisation suppose une approche participative et anticipatrice en opposition avec une perspective court-termiste et spéculative où toute forme de régulation est honnie. Elle suppose un projet commun partagé par des hommes et des femmes évoluant sur un territoire qui ne se réduit pas à un espace exclusivement économique mais devient un lieu de vie aux interactions nombreuses fondées sur des valeurs d'ordre éthique. Ainsi, ce qui peut expliquer la difficulté d'une GRH $\mathrm{T}$ est aussi ce qui montre son intérêt : il y a en germe dans cette notion, la possibilité d'incarner des interactions fécondes car guidées par le respect et la confiance mutuels. Deux dimensions qui, lorsqu'elles sont absentes, réduisent cette démarche d'anticipation à une planification laborieuse et sclérosante. Là est sans doute le défi à relever pour construire une GRH-T crédible : dans la capacité à rassembler l'ensemble des acteurs et à la fédérer autour d'un management durable de l'ensemble des ressources, humaines, technologiques, financières, économiques... de ce lieu de vie qu'est le territoire. 


\section{Références :}

Becattini G. (1992) Le district marshallien : une notion socio-économique, in Benko G. et Lipietz A., Les régions qui gagnent, PUF.

Berger G. (1964). Phénoménologie du temps et Prospective, PUF.

Blanchet A, Gotman A. (2010), L'enquête et ses méthodes - L'entretien, A. Colin, Parsi, $2^{\text {ème }}$ éd.

Bories-Azeau I., Loubes A. (2009). Les maisons de l'emploi, un dispositif en faveur d'une gestion territoriale des ressources humaines? XXème congrès de l'AGRH, Saint Malo.

Bories-Azeau I., Loubes A. Estève J.M . (2008). Emergence d'une GRH territoriale et réseau inter firmes. XIXème Congrès de l'AGRH, Dakar.

Boyer L., Scouarnec A.(2009). La Prospective des Métiers. Editions EMS.

Bres L.; Raufflet E. (2013), Pluralistic organizations in management: One phenomenon and multiple theoretical developments, AOM Annual Conference, Orlando, Florida, 2013

Coghlan D., Brannick T. (2005), Doing Action Research in your Own Organization (2nd Ed.) London: sage

Crozier M., Friedberg E. (1977). L'acteur et le système Ed. Du Seuil, Paris.

Culié J.D., Defélix C., Retour D., Valette A. (2006). Les pôles de compétitivité, laboratoires d'innovation en ressources humaines? XVIIème Congrès de l'AGRH, Reims.

Dubet F. (2002). Le déclin de l'institution. Paris, Ed. Du Seuil.

Eden C., Huxham C. (1996), «Action research for management research », British Journal of Management, Vol. 7, $\mathrm{N}^{\circ} 1$, pp.75-86

Friedberg E. (1993). Le pouvoir et la règle. Dynamique de l'action collective organisée. Ed. Du Seuil. Coll. Essais.

Fromentin J.C. (2012). Le temps des territoires. Pour un nouveau modèle de croissance. François Bourin Editeur.

Gazier B. (2008). «Flexicurité et marchés transitionnels du travail: esquisse d'une réflexion normative », Travail et Emploi, n¹13, p.117 à 127.

Granovetter M. (1973). "The strength of weak ties", American Journal of Sociology, LXXVIII, 6, PP1360-1380.

Granovetter M. (1985) "Economic Action and Social Structure: The problem of Embeddedness". American Journal of Sociology 91 (3): 481.

Granovetter M. (1990). "The Old and the New Economic Sociology", in Friedland R., Robertson A., Beyond the market, (Ed.) Aldine, New York.

Guthmann JF., Biehler M., Palasz JP, (2010), «La mise à disposition de personnel entre membres d'un pôle de compétitivité. Evaluation d'une expérimentation législative », Rapport IGAS et CGEF, avril. 
Habermas, J. (1981) Théorie de l'agir communicationnel. Rationalité de l'agir et rationalisation de la société. Tome 1. Pour une critique de la raison fonctionnaliste. Tome 2. Fayard, Coll. L'espace du politique (1987 pour la traduction française).

Kaisergrüber D. (2006). Flexi-sécurité : L'invention des transitions professionnelles, Editions de l'ANACT.

King N. (2004), "Using interviews in qualitative research" in C.Cassel and G.Symon (eds) Essential Guide to qualitative methods in Organizational Research. London, Sage, pp.11-22

Marshall A. (1890), Principles of Economics, Macmillan, traduction française : Principes d'économie politique, Giard et Brière, 1906.

Mucchielli A. (2009), Dictionnaire des méthodes qualitatives en sciences humaines et sociales, A. Colin, Paris, $2^{\text {ème } e ́ d . ~}$

Pesqueux Y. (2007) Gouvernance et privatisation, coll « la politique éclatée », PUF, Paris.

Polanyi K. (1944). La grande transformation. Aux origines politiques et économiques de notre temps. Ed Gallimard, 1983, pour la traduction française.

Retour D . (2008) (coord.). Dossier «Pôles de compétitivité », Revue Française de Gestion, №190, 2008/10, pp. 93-209.

Robson C., (2002), Real World Research (2 ${ }^{\text {nd }}$ Ed.), Oxford: Blackwell. 